\title{
Therapy for Chronic Recurrent Osteomyelitis with Multi-Resistant Pseudomonas aeruginosa Using Local Antibiotic Release by a Polymethylmethacrylate Custom-Made Tibia Nail
}

\author{
Debora Senn, Sebastian Gehmert, ${ }^{2}$ Peter E. Ochsner, ${ }^{3}$ Klaus-Dieter Kühn, ${ }^{4}$ and Andrej M. Nowakowski ${ }^{2}$
}

\begin{abstract}
Background: The treatment of chronic osteomyelitis by antibiotics administered intravenously is limited by the blood supply that is not present in bone sequestra. Local delivery of antibiotics using acrylic bone cement has been reported with favorable results for treatment of chronic osteomyelitis.

Case Presentation: Treatment of a patient with recurrent osteomyelitis after a third- degree open tibia fracture at our hospital failed several times because of recurrent infection with Pseudomonas aeruginosa that later became multi-resistant. Suitable antibiotic treatment was limited to colistin that was mixed with polymethylmethacrylate (PMMA) matrix (Copal ${ }^{\circledR} \mathrm{G}+\mathrm{C}$ Plus, Heraeus, Hanau, Germany) and used to cover an Ender nail. The PMMAtibia nail construct was placed intra-medullary for two weeks under appropriate antibiotics applied intravenously. The patient has been pain free for 18 months without any signs of infection and with normal blood tests.

Conclusion: We provide evidence that colistin can be applied locally with Copal $\mathrm{G}+\mathrm{C}$ providing appropriate concentration because of elution over a period of at least two weeks. This technique demonstrates a custom-made solution for patients with multi-resistant osteomyelitis for $P$. aeruginosa when conventional treatment fails.
\end{abstract}

Keywords: colistin; Pseudomonas aeruginosa; osteomyelitis; Copal ${ }^{\circledR} \mathrm{G}+\mathrm{C}$; polymethylmethacrylate

C HRONIC OSTEOMYELITIS occurs frequently after direct contamination from open fracture, surgery, or as a result of spread from other sources of infection. The persistence of micro-organisms and a low-grade inflammation define chronic osteomyelitis that can be associated with fever and bone destruction [1]. Bone segments with necrosis that are separated from the blood supply cannot be targeted by antibiotics or inflammatory cells and provide a satisfying matrix for bacterial retreat. Thus, local delivery of antibiotics using acrylic bone cement has been reported with favorable results for treatment of chronic osteomyelitis [2].

Polymethylmethacrylate (PMMA) is widely used as a local drug delivery system providing initially high local concen- trations without exceeding toxic serum concentrations However, commercially available antibiotic-impregnated cement provides only a fixed level of antibiotics because specific antibiotics interfere with cement components and/or the curing process and some antibiotics are not heat-stable. The mechanic resistance of PMMA is altered by adding antibiotics and can decrease especially if the amount of antibiotics within the powder exceeds $10 \%$ of the total volume [3]. Moreover, each antibiotic will have a different impact on the mechanic stability of the cement and has to be investigated prior to application if not provided by manufacturer or data from literature and if additional mechanical stability is required for healing. Each antibiotic will have different

\footnotetext{
${ }^{1}$ Department of Surgery, Kantonsspital Olten, Olten, Switzerland.

${ }^{2}$ Department of Orthopedics and Trauma Surgery, University of Basel, Basel, Switzerland.

${ }^{3}$ Interdisciplinary Unit for Orthopaedic Infections and Clinic for Orthopaedics and Trauma Surgery, Kantonsspital Baselland Liestal, Liestal, Switzerland.

${ }^{4}$ Department of Orthopedics, University Hospital Graz, Graz, Austria.

(C) Debora Senn et al. 2017; Published by Mary Ann Liebert, Inc. This Open Access article is distributed under the terms of the Creative Commons License (http://creativecommons.org/licenses/by/4.0), which permits unrestricted use, distribution, and reproduction in any medium, provided the original work is properly credited.
} 
effects on elution properties and can interfere with elution efficacy [4].

Colistin-loaded bone cement has been used frequently for total joint replacement but only prophylactically because appropriate colistin elution of the only commercially available cement is limited to $72 \mathrm{~h} \mathrm{[5].} \mathrm{We} \mathrm{present} \mathrm{a} \mathrm{case} \mathrm{of} \mathrm{osteomy-}$ elitis with multi-drug-resistant Pseudomonas aeruginosa that required a custom-made colistin-laden bone cement spacer stabilized with an Ender nail.

\section{Case Presentation}

A 49-year-old man was admitted to our department because of a motor bike accident with a third-degree open contaminated tibial fracture ( $\mathrm{AO} 42 \mathrm{C}$ ). Initial treatment was external fixation, vigorous debridement, lavage of the wound, and negative pressure dressings. Pseudomonas aeruginosa was detected in two of three tissue biopsies harvested during subsequent debridements. Antibiotic therapy comprised cefepime and obracin for two weeks and the remaining soft tissue defect was covered with a free scapula flap followed by a plate osteosynthesis of the tibia fracture. The patient was discharged with oral antibiotics (ciprofloxacin) and unremarkable wounds. Two months later the patient was readmitted with an implant failure fracture caused by a fall and osteosynthesis material was removed. Noteworthy, four of five biopsies were positive for coagulase-negative staphylococci (CoNS) resistant to methicillin that required two weeks of treatment with vancomycin. The fracture was stabilized with a re-amed static interlocking nail and oral antibiotic treatment was continued with ciprofloxacin (for P. aeruginosa) and rifampicin (for CoNS) for six months. Follow-up radiographs showed no fracture consolidation and the tibia nail was dynamized to provide an appropriate stimulus for fracture healing. However, the patient complained of increased pain levels and a limb shortening of $15 \mathrm{~mm} 10 \mathrm{mo}$ after revision with no radiographic signs of fracture consolidation. The nail was removed 20 mo after last fracture sur- gery and the intra-medullary space was re-amed. An external fixateur was applied to restore leg length by distraction. Five of six biopsies revealed $P$. aeruginosa and intravenous antibiotic treatment was established for two weeks. Afterwards, the tibia was stabilized with a $\mathrm{T} 2^{\mathrm{TM}}$ recon nail (Stryker Switzerland, Selzach, Switzerland) and the remaining bone defect was bridged with a vascularized bone graft from the medial femoral condyles combined with cancellous autograft. The patient was able to walk with full weight bearing six months after revision surgery.

The patient presented four years later with a fistula (Fig. 1) and oral antibiotic treatment was started with ciprofloxacin (500 mg twice per day) and was discontinued because of a multi-drug-resistant $P$. aeruginosa that required removal of the osteosynthesis material. A multi-drug-resistant $P$. aeruginosa was confirmed in four of four tissue biopsies as well as by sonication of the osteosynthesis material. After surgery, antibiotic therapy was initiated with intravenously administered piperacillin/tazobactam (four doses of $4 \mathrm{~g} / \mathrm{d}$ ) for one week and switched to imipenem (four doses of $500 \mathrm{mg} / \mathrm{d}$ ) and tobramycin $(480 \mathrm{mg} / \mathrm{d})$ for the following six weeks. Local antimicrobial therapy was started four weeks after osteosynthesis removal by implantation of a custom-made PMMA/Ender nail construction using $\mathrm{Copal}^{\circledR} \mathrm{G}+\mathrm{C}$ Plus (Heraeus, Hanau, Germany) supplemented with colistin. Copal $\mathrm{G}+\mathrm{C}$ is based on Palacos ${ }^{\circledR} \mathrm{R}+\mathrm{G}$ (Heraeus) but also contains clindamycin. In vitro experiments were performed prior to implantation to evaluate the impact of added colistin on elution efficacy and mechanical properties becaues PMMA cement with a mixture of gentamicin, clindamycin, and colistin is currently not available commercially.

Palacos $\mathrm{R}+\mathrm{G}$ supplemented with colistin was investigated according to ISO 5833 (2002) and DIN 53435 (1984, bending strength, impact strength). Elution of colistin was determined by using cylindrical PMMA samples (diameter $2 \mathrm{~cm}$, height $1 \mathrm{~cm}$, surface area $17.66 \mathrm{~cm}^{2}$ ) and high-performance liquid chromatography tandem mass spectrometry (HPLC-MS/MS) analysis. Mechanical properties were associated with the
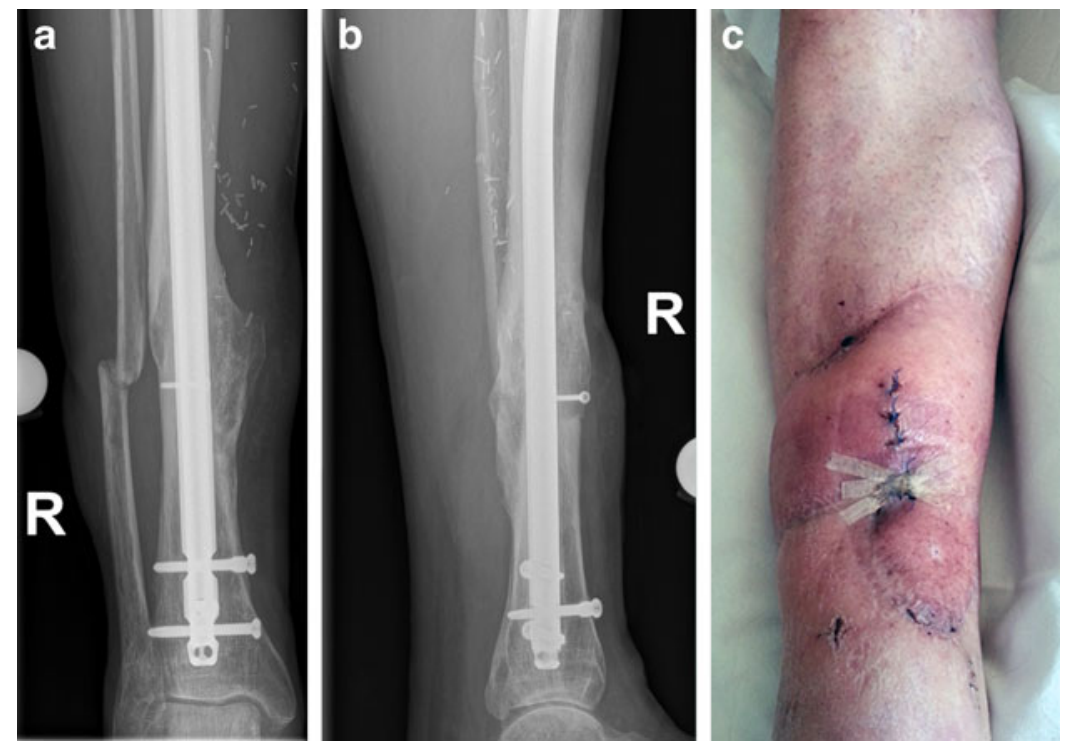

FIG. 1. Four years after the last surgery the patient presented a bridged non-union (a, b) but with a fistula at one of the margins of the earlier free scapula flap (c). 

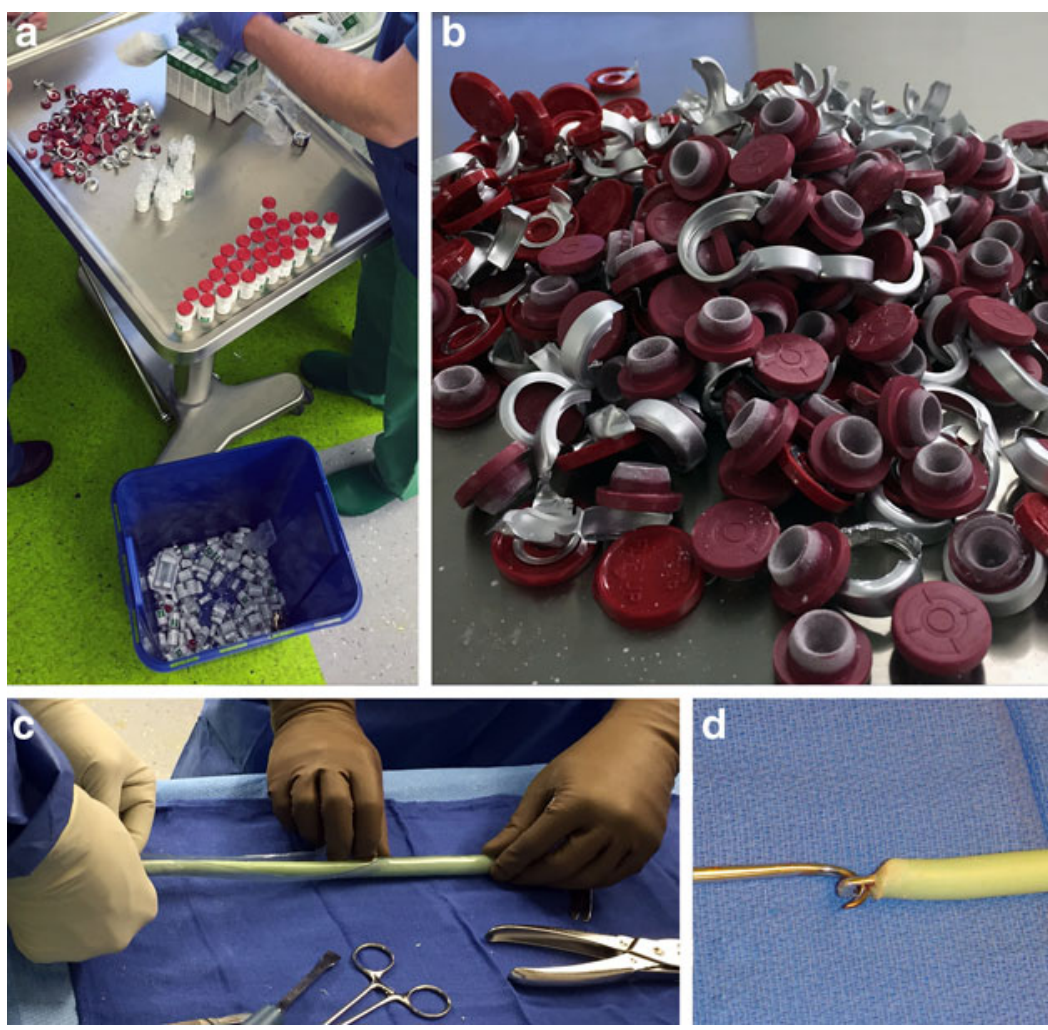

FIG. 2. Colistin is available in Switzerland only in vials of $1 \times 10^{6} \mathrm{IE}$, which corresponds to $33.3 \mathrm{mg}$ colistin. Thus, 60 vials of $1 \times 10^{6} \mathrm{IE}$ colistin had to be used to provide the required amount of colistin (a, b). The mix of Copal ${ }^{\circledR} \mathrm{G}+\mathrm{C}$ (Heraeus, Hanau, Germany) and colistin (Copal ${ }^{B} \mathrm{G}+\mathrm{C}$ Plus; Heraeus) was infused in to the plastic sleeve holding the Ender nail to provide a stable, even coating of the nail (c). The nail was placed into the intra-medullary space after reaming and removed after two weeks $(\mathbf{d})$.

amount of added colistin whereas two grams of colistin showed only a small reduction of ISO and DIN mechanical properties of the tested PMMA cement. In contrast, five grams of colistin led to a substantial reduction of mechanical properties.

The PMMA coating of the nail was prepared first by mixing two grams of colistin with $40 \mathrm{~g}$ Copal $\mathrm{G}+\mathrm{C}$ referred to as Copal $\mathrm{G}+\mathrm{C}$ Plus based on in vitro data that showed an appropriate release of colistin. Colistin is available in Switzerland only in vials of $1 \times 10^{6} \mathrm{IE}$, which corresponds to $33.3 \mathrm{mg}$ colistin. Thus, 60 vials of $1 \times 10^{6} \mathrm{IE}$ colistin were used to provide the required amount of colistin. The Ender nail was then placed in a plastic sleeve and the mix of Copal $\mathrm{G}+\mathrm{C}$ plus was infused into the plastic sleeve holding the Ender nail to provide a complete, uniform coating of the nail (Fig. 2a).

The PMMA-nail construct was placed into the intramedullary space (Fig. 3) and was removed after two weeks (Fig. 2b and 2c). All surgical procedures were performed by A.M.N. The patient was discharged with a pump to provide continuous intravenous antibiotic therapy with Tazobac ${ }^{\circledR}$ (Pfizer AG, Zürich, Switzerland) for an additional period of three months $(12 \mathrm{~g} / \mathrm{d})$. No side effects were observed during the two weeks of local antibiotic therapy.

Leucocyte counts and C-reactive protein level normalized within two weeks after PMMA-nail construct removal. After the end of the three months of continuous intravenously applied Tazobac therapy no further antibiotic treatment was needed until the follow-up in 2017. The patient remains pain free without antibiotics, can walk without assistance and limping, and presents no signs of infection (Fig. 4).

\section{Discussion}

Several PMMA cement products with more than one antibiotic such as Copal $\mathrm{G}+\mathrm{C}$ (containing one gram of gentamicin and one gram of clindamycin in $40 \mathrm{~g}$ cement) are available commercially. However, for the present case the antibiotic-laden bone cement (ALBC) required colistin because of the multi-resistant $P$. aeruginosa. Gasparini et al. [6] showed that elution of colistin from PMMA discontinued its elution after seven days when using $0.96 \mathrm{~g}$ colistin per $40 \mathrm{~g}$ cement $(2.4 \%$ colistin) whereas $0.6 \%$ colistin formulation ( $0.24 \mathrm{~g}$ per $40 \mathrm{~g}$ PMMA) already discontinued after the first hour. These results are in agreement with a study by Wahlig et al. [7] that reported decreasing colistin release from plain Palacos ${ }^{\circledR} \mathrm{R}$ (Heraeus) for the first four days when using $0.5 \mathrm{~g}$ of antibiotic. However, no data have been published so far on the elution of colistin and possible interaction when added to Copal G + C (based on Palacos R+G).

Sustained high antibiotic release is desired because antibacterial activity is concentration-dependent and will not continue once release rates decrease below a certain level which is associated with inducing antibiotic resistance. Our data are in agreement with a previous reported case for which a high-dose colistin- and 


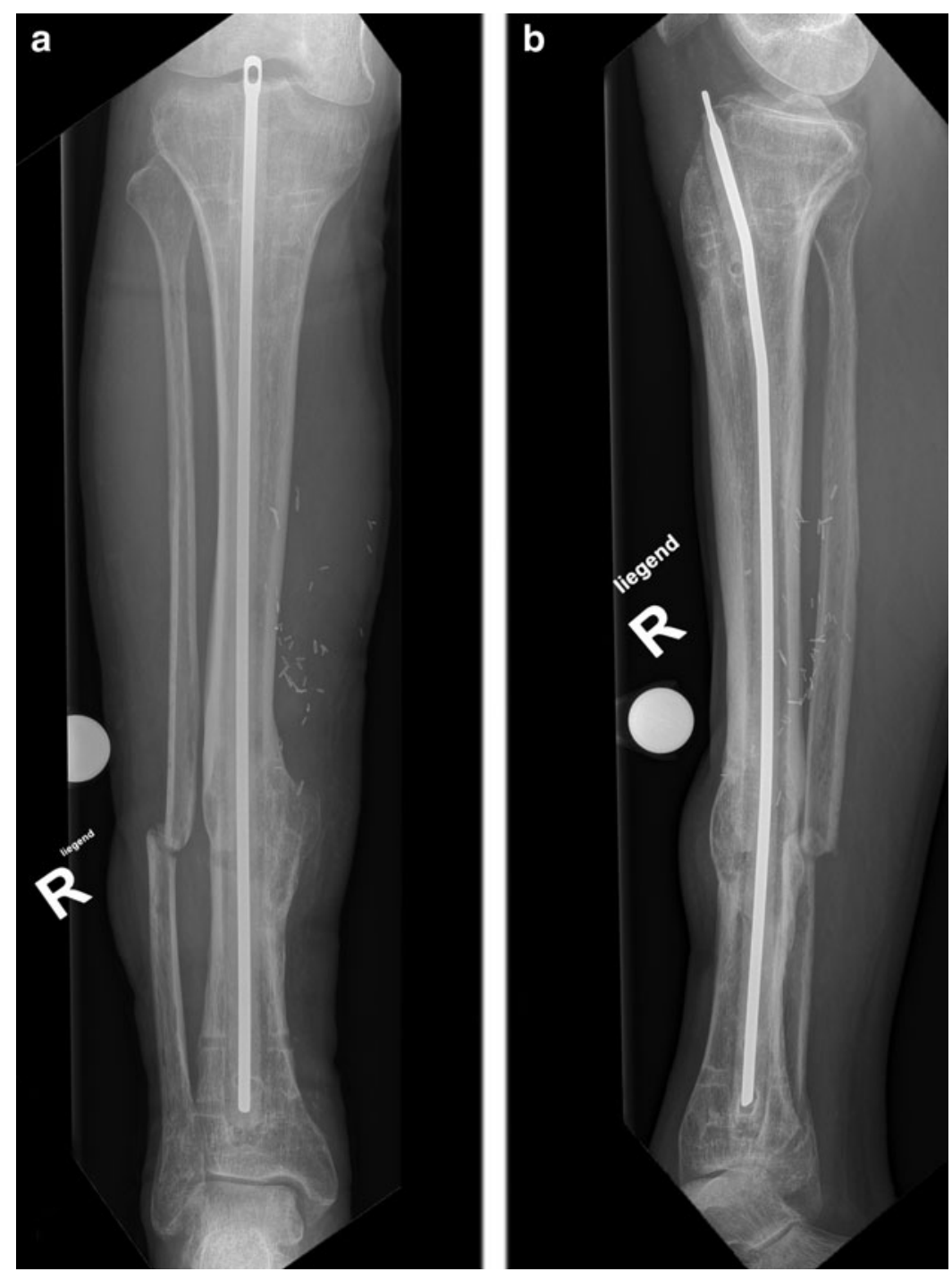

FIG. 3. The Ender nail covered with Copal ${ }^{\circledR} \mathrm{G}+\mathrm{C}$ Plus (Heraeus, Hanau, Germany) was placed into the intra-medullary space for two weeks. Radiographs shows an intra-medullary Ender nail in an anteroposterior (a) and lateral (b) view.
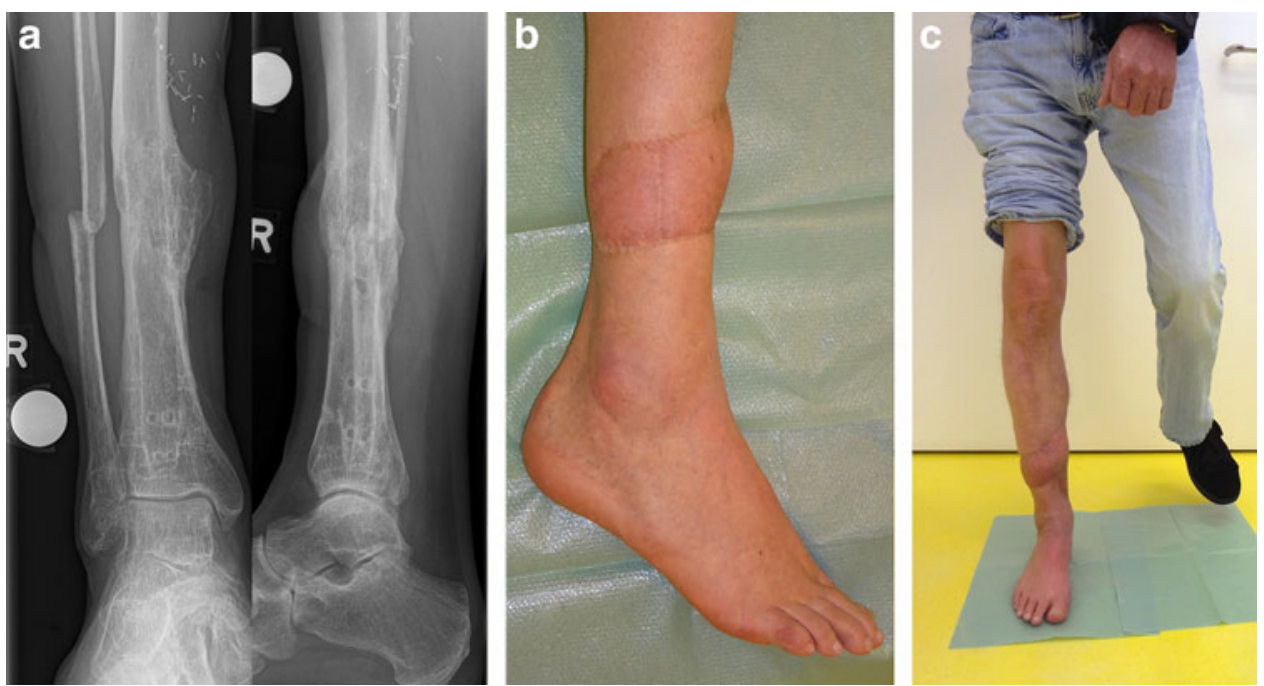

FIG. 4. One and a half years after removal of the Ender nail the tibia was stable (a) and the soft tissues showed no signs of infection $(\mathbf{b}, \mathbf{c})$. 
tobramycin-impregnated cement was used for $P$. aeruginosa treatment [8].

The quantity of released antibiotic depends directly on the surface of the ALBC. Thus, the antibiotic release could be higher for a structured surface of the ALBC instead of the smooth-surface ALBC used in our case (Fig. 3) [9].

A recent study provides evidence that several antibiotics including colistin decrease cell viability and osteogenic activity [10]. For vancomycin, tobramycin. or amikacin this is only true for high concentrations. Therefore, the local risks and adverse effects of colistin on bone or soft tissue require supplementary studies.

What part the local administration of colistin played in the final treatment of our case is left to speculation because at the same time another intra-medullary reaming could have removed causative sequestra possibly allowing the additional systemic antibiotic treatment with Tazobac over three months to eliminate the bacteria.

\section{Conclusion}

We provide evidence that colistin can be locally applied with Copal $\mathrm{G}+\mathrm{C}$ providing appropriate concentration because of elution over a period of at least two weeks. This technique demonstrates a custom-made solution for patient with multi-resistant osteomyelitis for $P$. aeruginosa when conventional treatment fails.

\section{Acknowledgments}

No financial support was received for this study.

All procedures performed in studies involving human participants were in accordance with the ethical standards of the institutional and/or national research committee and with the 1964 Helsinki Declaration and its later amendments or comparable ethical standards. For this type of study formal consent is not required.

\section{Author Disclosure Statement}

The authors have no conflicts of interest to declare.

\section{References}

1. Lew DP, Waldvogel FA. Osteomyelitis. N Engl J Med 1997;336:999-1007.

2. Anagnostakos K, Wilmes P, Schmitt E, Kelm J. Elution of gentamicin and vancomycin from polymethylmethacrylate beads and hip spacers in vivo. Acta Orthop 2009;80:193-197.

3. Hendrich C, Frommelt L, Eulert J. Septische Knochen-und Gelenkchirurgie. Springer Berlin Heidelberg, 2004.

4. Kühn K-D: PMMA Cements. Springer Berlin Heidelberg, 2014.
5. Ruzaimi MY, Shahril Y, Masbah O, et al. Antimicrobial properties of erythromycin and colistin impregnated bone cement. An in vitro analysis. Med J Malaysia 2006;61(Suppl A):21-26.

6. Gasparini G, De Gori M, Calonego G, et al. Drug elution from high-dose antibiotic-loaded acrylic cement: A comparative, in vitro study. Orthopedics 201437:e999-1005.

7. Wahlig H, Dingeldein E. Antibiotics and bone cements. Experimental and clinical long-term observations. Acta Orthop Scand 1980;51:49-56.

8. Krajewski J, Bode-Böger SM, Tröger U, et al. Successful treatment of extensively drug-resistant Pseudomonas aeruginosa osteomyelitis using a colistin- and tobramycinimpregnated PMMA spacer. Int J Antimicrob Agents 2014; 44:363-366.

9. Moojen DJF, Hentenaar B, Charles Vogely H, et al. In vitro release of antibiotics from commercial PMMA beads and articulating hip spacers. J Arthroplasty 2008;23:11521156.

10. Rathbone CR, Cross JD, Brown KV, et al. Effect of various concentrations of antibiotics on osteogenic cell viability and activity. J Orthop Res 2011;29:1070-1074.

Address correspondence to: PD Dr. Dr. Dipl-lng. (FH) Andrej M. Nowakowski Department of Orthopedics and Trauma Surgery

University of Basel Spitalstrasse 21 4056 Basel Switzerland

E-mail: andrej.nowakowski@unibas.ch

$\begin{aligned} & \text { Abbreviations Used } \\ & \mathrm{ALBC}= \text { antibiotic-loaded bone cement } \\ & \mathrm{CoNS}= \text { coagulase negative staphylococci } \\ & \mathrm{HPLC}-\mathrm{MS} / \mathrm{MS}= \text { high-performance liquid chromatography } \\ & \text { tandem mass spectrometry } \\ & \mathrm{PMMA}=\text { polymethylmethacrylate }\end{aligned}$

Cite this article as: Senn D, Gehmert S, Ochsner PE, Kühn K-D, Nowakowski AM (2017) Therapy of chronic recurrent osteomyelitis with multi-resistant Pseudomonas aeruginosa using local antibiotic release by a polymethylmethacrylate custom-made tibia nail. Surgical Infections Case Reports 2:1, 26-30, DOI: 10.1089/ crsi.2017.0005 